\title{
EFEITO DO PRÉ-TRATAMENTO ULTRASSÔNICO SOBRE A CINÉTICA DE LIOFILIZAÇÃO E PROPRIEDADES DE REIDRATAÇÃO DE JAMELÃO (Syzygium cumini)
}

\author{
R. J. BRANDÃO*, D. S. ANDRADE, M. M. PRADO, L. G. MARQUES, \\ Universidade Federal de Sergipe, Departamento de Engenharia Química \\ *e-mail: rodolfoj.brandao@hotmail.com
}

\begin{abstract}
RESUMO
O jamelão (Syzygium cumini) é uma fruta tropical, rica em compostos antioxidantes, mas altamente perecível. A liofilização é uma excelente alternativa para estender a vida de prateleira do jamelão e facilitar sua comercialização, além de assegurar a conservação e a disponibilidade de seus compostos bioativos em produtos de maior valor agregado. Esta técnica apresenta, no entanto, alto custo operacional, devido ao longo tempo do processo, havendo a necessidade de assistida por diferentes pré-tratamentos para aumentar as taxas de secagem. Logo, o objetivo do presente trabalho foi avaliar o potencial uso do pré-tratamento ultrassônico para acelerar o processo de liofilização de jamelão, bem como para melhorar suas características de reidratação. Antes da liofilização, o material foi submetido a um banho ultrassônico, com frequências de 25 e $40 \mathrm{kHz}$, e tempos de exposição de 3 e $10 \mathrm{~min}$. O processo de liofilização foi conduzido a $98 \mu \mathrm{mHg}$ e a $-50^{\circ} \mathrm{C}$. A aplicação da sonicação provocou um aumento na difusividade efetiva de 30 a $46 \%$, intensificando assim as taxas de secagem. As amostras pré-tratadas tiveram um aumento em torno de $65 \%$ na razão de reidratação na saturação. Em relação à amostra sem tratamento, o material sonicado a $40 \mathrm{kHz}$, durante $10 \mathrm{~min}$, apresentou uma melhora na capacidade de absorver água de, aproximadamente, $141 \%$ e uma retenção de cerca $82 \%$ dos sólidos solúveis, o que resultou no maior índice de capacidade de reidratação.
\end{abstract}

\section{INTRODUÇÃO}

Jamelão (Syzygium cumini) é uma fruta típica de regiões tropicais, conhecida por seu elevado teor de vitaminas, sais minerais e compostos antioxidantes. Por conta de sua elevada perecibilidade, torna-se necessária a utilização de métodos de preservação que minimizem o uso de conservantes e preservem as características nutricionais desta fruta.

As frutas vêm ganhando grande atenção no que diz respeito a utilização de técnicas de beneficiamento, tornando-as aptas ao consumo humano, mesmo após longos períodos de estocagem (LAGO et al., 2006; MARQUES et al., 2007; SOGI; et al., 2014).

Com o propósito de diminuir perdas durante o transporte e estocagem, diversos métodos de secagem foram, ao longo do tempo, otimizados com o intuito de reduzir os gastos energéticos do processo, garantindo, desta forma, que o produto final chegue ao consumidor com melhor qualidade e menor custo. Dentre os diversos métodos de secagem utilizados no contexto atual, têm-se, como expoentes, a secagem convectiva, 
infravermelho, leito fluidizado e a liofilização (MARQUES et al., 2006).

A secagem por convecção forçada envolve a passagem de ar quente pelo material. Embora seja a técnica mais empregada para diferentes materiais, esta envolve alto consumo energético, devido à baixa eficiência, e requer longos tempos de secagem, o que geralmente compromete a qualidade de materiais termossensíveis (SANTOS et al., 2011).

Segundo Ratti (2001), a liofilização é uma técnica de secagem que retira a umidade contida no material através do congelamento da parte líquida e posterior sublimação do gelo. Por trabalhar com baixas temperaturas e, geralmente, sob vácuo, este processo tem, como uma de suas principais características, a minimização de mudanças estruturais, além de contribuir na preservação de constituintes termossensíveis, como materiais biológicos (fungos e enzimas), farmacêuticos (antibióticos e vacinas), alimentos (frutas, sucos e carnes) e produtos químicos; gerando produtos de qualidade superior quando comparados às outras técnicas de secagem (MARQUES, 2008).

Por esta técnica requerer longos períodos de secagem, elevando, assim, o custo do processo, a utilização de pré-tratamentos vem sendo largamente empregada nas indústrias. De acordo com Jambrak et al., (2006) e Mason (1998), a energia proveniente de ondas ultrassônicas vem sendo utilizada nos processos de secagem, uma vez que as amplitudes das vibrações causadas por essas ondas são capazes de aumentar a transferência de calor e de massa, por produzirem mudanças em gradientes de concentração e nos coeficientes de difusividade dos materiais.

Segundo Keymak-Ertekin (2002), o processo de secagem pode afetar a estrutura do material e alterar as propriedades osmóticas das paredes celulares, fazendo com que a capacidade de reidratação seja considerada uma forma de medida de danos.

Assim, o objetivo deste trabalho foi analisar o fenômeno de transferência de massa durante $o$ processo de liofilização e reidratação de jamelão, com e sem a utilização de pré-tratamento ultrassônico.

\section{MATERIAIS E MÉTODOS}

Os estudos foram realizados no Laboratório de Fenômenos de Transporte em Sistemas Particulados do Departamento de Engenharia Química e no Laboratório de Análise de Compostos Orgânicos Poluentes do Departamento de Química da Universidade Federal de Sergipe.

\subsection{Materiais}

O material utilizado nesta pesquisa foi o jamelão (Syzygium cumini), oriundo do mercado de Aracaju.

\subsection{Metodologia experimental}

\subsubsection{Caracterização do material}

Foram utilizados, em cada teste, cerca de $20 \mathrm{~g}$ de jamelão, representando, aproximadamente, 4 unidades desta fruta. Foram selecionados frutos de mesmo tamanho, visando assegurar a homogeneidade da amostra. Para tanto, suas dimensões (diâmetros máximo e mínimo) foram medidas por meio de um um paquímetro digital (Digimess, precisão de $10^{-2} \mathrm{~mm}$ ), sendo utilizados frutos com diâmetro médio de $10,98 \mathrm{~mm}$.

\subsubsection{Pré-tratamento Ultrassônico}

As frutas caracterizadas foram dispostas em béqueres de $500 \mathrm{ml}$, preenchidos com água destilada, e introduzidos no banho ultrassônico (UltraSonic Cleaner, Unique). Foram utilizadas quatro condições diferentes para esse tratamento; são elas: (a) frequência 
de $25 \mathrm{kHz}$ durante 3 minutos, (b) $25 \mathrm{kHz}$ em 10 minutos, (c) $40 \mathrm{kHz}$ em 3 minutos e (d) 40 $\mathrm{kHz}$ em 10 minutos.

\subsubsection{Liofilização}

As amostras pré-tratadas foram acondicionadas em placas de Petri e armazenadas num congelador, juntamente com amostras que não foram imersas no banho ultrassônico, chamadas amostras Controle.

O liofilizador (Liobras L101, Liotop) foi ligado e, ao atingir a temperatura de, aproximadamente, $-50^{\circ} \mathrm{C}$, as amostras foram retiradas do congelador e inseridas rapidamente nas prateleiras presentes no interior da câmara de acrílico do liofilizador. A bomba de vácuo foi, então, ligada. A partir desse momento, o processo de liofilização teve início, com o valor de pressão, mostrado no painel, atingindo a marca de $98 \mu \mathrm{mHg}$.

Em tempos pré-determinados, a bomba era desligada, as amostras eram retiradas do liofilizador e pesadas na balança. Logo após, as mesmas eram colocadas de volta no equipamento, dando-se continuidade ao processo. A partir da constatação de que a massa não mais variava significativamente com o tempo, o processo de secagem foi interrompido e o equipamento desligado.

\subsubsection{Reidratação}

Após as amostras serem liofilizadas, fez-se a reidratação das mesmas. Com o uso de um béquer preenchido com água destilada, à temperatura ambiente, inseriu-se a amostra e, de tempos em tempos, a mesma era retirada do béquer, com o excesso de água sendo removido com o auxílio de papel toalha para a aferição de sua massa. Esse procedimento foi repetido até que a massa da amostra não mais variasse com o tempo, ou quando começasse a perder massa, indicando que a estrutura da fruta havia sido danificada. A cinética de reidratação foi analisada em termos da evolução ao longo do tempo do processo da razão de reidratação (RR) como apresentada na Equação 1:

$$
\text { R.R. }=\frac{X_{(t)}}{X_{0}}
$$

Foram calculados, também, os índices de reidratação de Lewick, que são: capacidade de absorção de água (CAA), capacidade de retenção de massa seca $\left(\mathrm{CRM}_{\mathrm{S}}\right)$ e a capacidade de reidratação $(\mathrm{CR})$.

$\mathrm{O}$ índice CAA, proposto por Lewick (1998), calculado a partir da Equação 2, fornece informação sobre a capacidade de absorção de água do material seco:

$$
C A A=\frac{m_{r h}\left(100-S_{r h}\right)-m_{d}\left(100-S_{d}\right)}{m_{0}\left(100-S_{0}\right)-m_{d}\left(100-S_{d}\right)}
$$

Sendo $m$ a massa, $S$ o teor de sólido seco e os subscritos $0, d$ e $r h$ referem-se à amostra fresca, desidratada e reidratada, respectivamente. $\mathrm{O}$ índice varia de 0 a 1 . Quanto menor o índice, a capacidade de absorver água por parte da fruta fica mais prejudicada, impossibilitando a mesma de atingir o teor de umidade próximo da amostra fresca, evidenciando que o processo influenciou a estrutura do material.

$\mathrm{O}$ índice $\mathrm{CRM}_{\mathrm{s}}$, obtido a partir da Equação 3, mensura a perda de massa seca durante a reidratação, expressando a habilidade do material em reter sólidos solúveis (LEWICK, 1998).

$\mathrm{CRM}_{\mathrm{s}}=\frac{\mathrm{m}_{\mathrm{rh}} \mathrm{S}_{\mathrm{rh}}}{\mathrm{m}_{\mathrm{d}} \mathrm{S}_{\mathrm{d}}}$

$\mathrm{O}$ índice também varia de 0 a 1 . Quanto mais o tecido da fruta é danificado, menor é o índice, pois maior é a lixiviação de sólidos durante a reidratação.

Finalmente, a capacidade de reidratação, apresentado na Equação 4, se baseia na capacidade do material em absorver 
água e reter sólidos solúveis. Quanto maior o dano sofrido pela amostra, menor o índice.

$$
\mathrm{CR}=\mathrm{CAA} \cdot \mathrm{CRM}
$$

\subsubsection{Umidade}

A determinação de umidade dos frutos de jamelão "in natura", liofilizados e reidratados foi realizada pelo método de secagem em estufa (AL 102/42, AmericanLab) a $(105 \pm 3)^{\circ} \mathrm{C}$ por 24 horas, até a obtenção da massa de sólido seco, conforme recomendado pela norma $\mathrm{n}^{\circ}$ 4.5.1 do Instituto Adolfo Lutz (LUTZ, 1985).

\subsection{Tratamento e Análise dos Dados}

A partir dos resultados obtidos nos experimentos de secagem, as curvas que representam a cinética foram traçadas, relacionando o adimensional de umidade (X.R) em função do tempo de processamento.

Para representar a cinética de liofilização do jamelão, utilizou-se no presente trabalho o modelo difusivo. Para tanto, as seguintes hipóteses foram adotadas:

- Transferência de massa unidimensional e predominantemente difusiva.

- O teor de umidade na superfície da partícula atinge instantaneamente o valor de equilíbrio $\left(\mathrm{X}_{\mathrm{e}}\right)$.

- Sistema com encolhimento negligenciável.

- Processo isotérmico com a difusividade mássica independente da umidade. inicial;

- Distribuição uniforme de umidade

A solução aproximada, truncada no primeiro termo da série, é expressa da seguinte forma:

$$
X R=\frac{X-X_{e}}{X_{0}-X_{e}}=\operatorname{a} \cdot \exp \left[-D_{\text {eff }} \cdot\left(\frac{t}{R^{2}}\right)\right]
$$

Para descrever a cinética de reidratação, foi utilizada a Equação de Weibull (MACHADO et al. 1999), conforme apresentada abaixo:

$$
\mathrm{RR}=\mathrm{RR}_{\mathrm{e}}-\left(1-\mathrm{RR}_{\mathrm{e}}\right) \exp \left[-\frac{\mathrm{t}}{\beta}\right]^{\alpha}
$$

A estimação dos parâmetros das Equações (7) e (8) foi feita via regressão nãolinear pelo método dos mínimos quadrados utilizando o software Statistica $8^{\circledR}$. A qualidade dos ajustes foi avaliada com base no coeficiente de correlação $\mathrm{R}^{2}$.

\section{RESULTADOS E DISCUSSÕES}

Na Figura 1 são apresentados os resultados típicos do adimensional de umidade em função do tempo para o jamelão liofilizado, com e sem pré-tratamento ultrassônico.

Figura 1 - Adimensional de umidade em função do tempo de liofilização, para frutos de Jamelão, com e sem pré-tratamento ultrassônico.

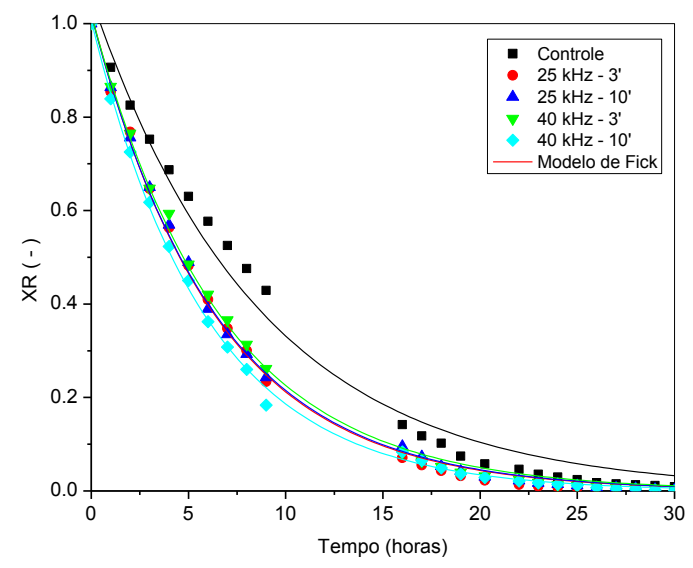

Ao se comparar, na Figura 1, o comportamento de secagem da amostraControle (sem pré-tratamento) com as amostras sonicadas, verifica-se que o prétratamento do jamelão acelerou a remoção de umidade no estágio inicial de secagem, 
correspondente às dez primeiras horas do processo. Além disto, a taxa de remoção de umidade neste período é intensificada na condição de maior frequência $(40 \mathrm{~Hz})$ e maior tempo de aplicação das ondas ultrassônicas (10 min).

Com base nos dados apresentados na Figura 1, verifica-se, ao se aplicar o prétratamento com ultrassom, uma redução de 99\% da umidade livre contida nos frutos de jamelão em um tempo $17 \%$ menor em relação àquele para a amostra-controle. Não houve, no entanto, uma influência significativa da frequência e do tempo de sonicação aplicados.

O comportamento de secagem do material sonicado e sem pré-tratamento foi analisado com base no modelo difusivo, cujos parâmetros estimados de sua solução simplificada são apresentados na Tabela 1.

Tabela 1 - Valores estimados dos parâmetros da solução simplificada do modelo difusivo aplicada à liofilização de Jamelão, com e sem prétratamento ultrassônico.

\begin{tabular}{lccc}
\hline Tratamento & $\mathrm{D}_{\text {eff }} \times 10^{9}\left(\mathrm{~m}^{2} / \mathrm{s}\right)$ & $\mathrm{a}(-)$ & $\mathrm{R}^{2}$ \\
\hline Controle & 3,869 & 1,056 & 0,989 \\
$25 \mathrm{kHz}-3$, & 5,256 & 1,021 & 0,998 \\
$25 \mathrm{kHz}-10$, & 5,193 & 1,018 & 0,999 \\
$40 \mathrm{kHz}-3$ & 5,033 & 1,020 & 0,998 \\
$40 \mathrm{kHz}-10$, & 5,637 & 1,006 & 0,999 \\
\hline
\end{tabular}

Ao se analisar a influência do banho ultrassônico sobre difusividade efetiva nos frutos de Jamelão ( $\mathrm{D}_{\text {eff }}$ ), a partir dos resultados apresentados na Tabela 1 , verificase que, com o uso do pré-tratamento, os valores de difusividade efetiva ( $\left.\mathrm{D}_{\text {eff }}\right)$ aumentaram de 30 a 46\%, em relação àquela da amostra-controle.

Logo, o pré-tratamento com ultrassom permitiu, por meio das vibrações causadas por essas ondas, alterar a estrutura do material, de modo a aumentar a difusividade efetiva de umidade, contribuindo para intensificar as taxas de transferência de massa durante a liofilização.
A Figura 2 mostra os resultados da razão de reidratação em função do tempo para o jamelão liofilizado, com e sem prétratamento ultrassônico.

Figura 2 - Razão de reidratação em função do tempo, para o jamelão liofilizado.

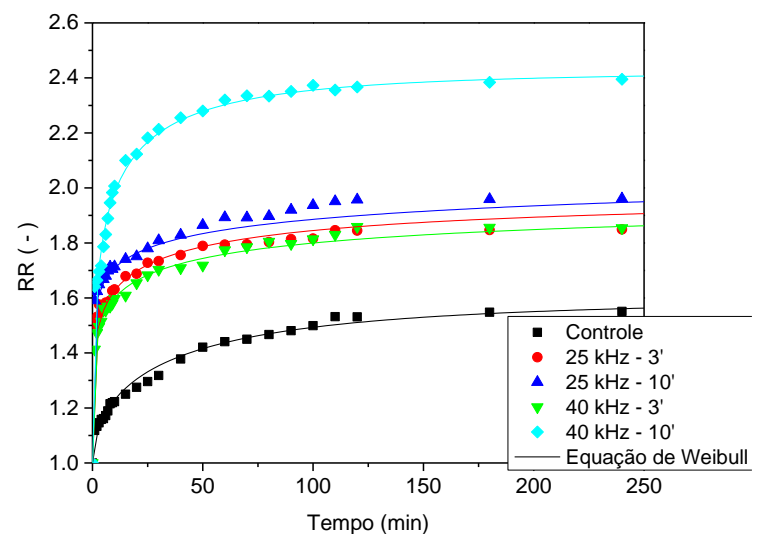

As amostras de jamelão sonicadas apresentaram os maiores valores de razão de reidratação durante todo $\mathrm{o}$ processo, comparadas à amostra-controle, conforme pode ser constatado na Figura 2. Para uma mesma frequência de onda ultrassônica verifica-se um aumento na razão de reidratação com o aumento do tempo de aplicação do pré-tratamento. Esse aumento foi mais significativo na amostra submetida à maior frequência $(40 \mathrm{~Hz})$.

A utilização do pré-tratamento com banho ultrassônico contribuiu efetivamente para melhorar as características de reidratação do jamelão. Isto devido à energia ultrassônica provocar cavitações internas no material, que levam a um aumento no número de poros, facilitando assim a transferência de massa durante a reidratação.

A aplicação do pré-tratamento ultrassônico a $40 \mathrm{kHz}$, durante $10 \mathrm{~min}$, resultou num aumento de quase $65 \%$ na razão de reidratação do material na saturação.

Na Tabela 2 são apresentados os valores dos parâmetros estimados da Equação de 
Weibull, utilizada para descrever a cinética de reidratação do jamelão liofilizado.

Com base nos elevados valores obtidos para o coeficiente de determinação e conforme mostrado na Figura 2, verifica-se a boa concordância entre os dados experimentais e aqueles preditos pela equação de Weibull.

Tabela 2 - Parâmetros estimados da equação de Weibull ajustada para o jamelão.

\begin{tabular}{lllll}
\hline Tratamento & $\mathrm{RR}_{\mathrm{e}}(-)$ & $\alpha(-)$ & $\beta(\min )$ & $\mathrm{R}^{2}$ \\
\hline Controle & 1,593 & 0,562 & 35,782 & 0,981 \\
$25 \mathrm{kHz}-3$, & 1,982 & 0,288 & 9,491 & 0,980 \\
$25 \mathrm{kHz}-10$ & 2,166 & 0,183 & 14,417 & 0,985 \\
$40 \mathrm{kHz}-3$, & 1,968 & 0,258 & 11,453 & 0,986 \\
$40 \mathrm{kHz}-10$, & 2,423 & 0,416 & 6,842 & 0,982 \\
\hline
\end{tabular}

$\mathrm{O}$ inverso do parâmetro $\beta$ pode ser comparado ao coeficiente de difusão efetivo do modelo de difusão, sendo que, quanto menor o valor desse parâmetro, mais rapidamente a fruta absorve água. De acordo com os resultados apresentados na Tabela 2, as taxas de reidratação das amostras liofilizadas pré-tratadas com ultrassom foram significativamente maiores que aquela da amostra-controle, conforme evidenciado pelos menores valores de $\beta$. Frutos de jamelão prétratados com ultrassom a $40 \mathrm{kHz}$ e durante 10 min tiveram a mais rápida absorção de umidade.

Na Tabela 3 são apresentados os índices de reidratação, que permitem avaliar o ganho de água (CAA.), a capacidade do material em reter sólidos solúveis $\left(\mathrm{CRM}_{\mathrm{s}}\right)$, e a capacidade do produto seco de se reidratar (CR).

Tabela 3 - Índices de reidratação do jamelão.

\begin{tabular}{llll}
\hline Tratamento & CAA & CRM $_{\mathrm{s}}$ & CR \\
\hline Controle & 0,078 & 0,818 & 0,064 \\
$25 \mathrm{kHz}-3$, & 0,121 & 0,801 & 0,089 \\
$25 \mathrm{kHz}-10$ & 0,187 & 0,673 & 0,123 \\
$40 \mathrm{kHz}-3$, & 0,116 & 0,778 & 0,093 \\
$40 \mathrm{kHz}-10$, & 0,271 & 0,423 & 0,119 \\
\hline
\end{tabular}

De acordo com os índices de Lewick calculados e apresentados na Tabela 3, é possível avaliar o efeito do pré tratamento ultrassônico sobre as propriedades de reidratação do jamelão. Verifica-se, de um modo geral, que o pré tratamento provocou uma diminuição no parâmetro $\mathrm{CRM}_{\mathrm{s}} \mathrm{e}$ elevação do CAA, demonstrando assim o aumento da capacidade de absorção da amostra e a intensificação do fenômeno de lixiviação da mesma.

Para frequências de 25 e $40 \mathrm{kHz}$, um maior período de aplicação do pré-tratamento ultrassônico (10 min) aumentou a capacidade de absorção de água do jamelão em 141 e $243 \%$, respectivamente, quando comparados àquela da amostra-controle. Entretanto, levou a perdas de sólidos solúveis entre 18 e $49 \%$. Maiores tempos tendem a facilitar a lixiviação, muito provavelmente devido ao "efeito esponja" causado pela sonicação.

A redução do tempo de sonicação para 3 minutos favorece a retenção de cerca 97, 6 e $95,1 \%$ dos sólidos solúveis, nas frequências de 25 e $40 \mathrm{kHz}$, aumentando em torno de $51 \%$ a capacidade de absorção de água.

\section{CONCLUSÕES}

A partir dos resultados obtidos no presente trabalho, conclui-se que as cinéticas de liofilização de jamelão, com e sem prétratamento ultrassônico, foram adequadamente representadas pelo modelo difusivo.

A influência do pré-tratamento ultrassônico sobre a transferência de massa na liofilização foi analisada em termos da difusividade mássica efetiva. A aplicação da sonicação resultou num aumento na difusividade efetiva do jamelão entre 30 e $46 \%$, em toda a faixa experimental investigada Isto devido a possíveis modificações estruturais no material causadas pelas ondas ultrassônicas. 
A utilização do pré-tratamento ultrassônico contribuiu, portanto, para intensificar as taxas de secagem, principalmente no início do processo de liofilização.

A sonicação dos frutos de jamelão antes da liofilização contribuiu também para aumentar a taxa de reidratação.

$\mathrm{O}$ aumento na frequência e no tempo de aplicação do banho ultrassônico melhorou a capacidade de absorção de água do produto liofilizado, mas levou a uma maior perda de sólidos solúveis.

Uma análise global do ganho de água e da retenção de matéria seca dos frutos liofilizados de jamelão indica que as condições ótimas para o pré-tratamento ultrassônico do material são uma frequência de $40 \mathrm{kHz}$ e período de aplicação de $10 \mathrm{~min}$. Nestas condições, em relação à amostra sem tratamento, houve uma melhoria na capacidade de absorver água de aproximadamente $141 \%$ e uma retenção de cerca $82 \%$ dos sólidos solúveis, o que resultou no maior índice de capacidade de reidratação

\section{NOMENCLATURA}

$\begin{array}{llr}\text { CAA } & \begin{array}{l}\text { Capacidade de } \\ \text { absorção de água } \\ \text { CR }\end{array} & \begin{array}{l}\text { Capacidade de reidratação } \\ \text { Capacidade de } \\ \text { retenção de massa } \\ \text { seca }\end{array} \\ \mathrm{CRM}_{\mathrm{s}} & {[-]} \\ \mathrm{D}_{\text {eff }} & \begin{array}{l}\text { Difusividade efetiva } \\ \text { Massa da amostra } \\ \mathrm{m}_{\mathrm{d}}\end{array} & \begin{array}{l}{[-]} \\ \text { desidratada }\end{array} \\ \mathrm{m}_{0} & \begin{array}{l}\text { Massa da amostra in } \\ \text { natura }\end{array} \\ \mathrm{m}_{\mathrm{rh}} & \begin{array}{l}\text { Massa da amostra } \\ \text { reidratada }\end{array} \\ \mathrm{R} & \begin{array}{l}\text { Raio da partícula } \\ \text { Adimensional de }\end{array} & {[\mathrm{g}]} \\ \mathrm{RR} & \begin{array}{l}\text { reidratação } \\ {[\mathrm{g}]}\end{array} \\ \end{array}$

\begin{tabular}{|c|c|c|}
\hline $\mathrm{RR}_{\mathrm{e}}$ & $\begin{array}{l}\text { Razão de reidratação } \\
\text { na saturação }\end{array}$ & \\
\hline $\mathrm{S}_{\mathrm{d}}$ & $\begin{array}{l}\text { Teor de sólidos da } \\
\text { amostra desidratada }\end{array}$ & $\begin{array}{r}\text { [g sólido } \\
\text { seco/g } \\
\text { amostra] }\end{array}$ \\
\hline $\mathrm{S}_{0}$ & $\begin{array}{l}\text { Teor de sólidos da } \\
\text { amostra in natura }\end{array}$ & $\begin{array}{r}\text { [g sólid } \\
\text { seco/ } \\
\text { amostra }\end{array}$ \\
\hline$S_{\text {rh }}$ & $\begin{array}{l}\text { Teor de sólidos da } \\
\text { amostra reidratada }\end{array}$ & $\begin{array}{l}\text { [g sólid } \\
\text { seco/ } \\
\text { amostra }\end{array}$ \\
\hline $\mathrm{t}$ & Tempo & {$[\mathrm{s} ; \mathrm{min} ; \mathrm{h}$} \\
\hline$X$ & $\begin{array}{l}\text { Teor de umidade em } \\
\text { base seca }\end{array}$ & $\begin{array}{r}\text { [g água/ } \\
\text { sólido seco }\end{array}$ \\
\hline X.R. & $\begin{array}{l}\text { Adimensional de } \\
\text { umidade }\end{array}$ & \\
\hline$\alpha$ & $\begin{array}{l}\text { Parâmetro de forma } \\
\text { do modelo de } \\
\text { Weibull }\end{array}$ & \\
\hline$\beta$ & Parâmetro de escala & {$[\mathrm{mil}$} \\
\hline
\end{tabular}

INSTITUTO ADOLFO LUTZ. Normas Analíticas do Instituto Adolfo Lutz. v. 1: Métodos químicos e físicos para análise de alimentos, 3. ed. São Paulo: IMESP, 1985. p. 23-5.

JAMBRAK, A. R.; MASON, T. J.; PANIWNYK, L.; LELAS, V. Accelerated drying of button mushrooms, Brussels sprouts and cauliflower by applying power ultrasound and its rehydration Properties. Journal of Food Engineering, v. 81, p. 88-97, 2007.

KAYMAK-ERTEKIN, F. Drying and rehydration kinetics of green and red peppers, Journal of Food Science, v. 67(1), p. 168175, 2002.

LAGO, E. S.; GOMES, E.; SILVA, R. Produção de geleia de jambolão (Syzygiu cumini Lamarck): Processamento, parâmetros físico-químicos e avaliação sensorial. Ciência 
e Tecnologia de Alimentos, v. 26, n. 4, p. 847-852, 2006.

LEWICKI, P. P. Some remarks on rehydration of dried foods, Journal of Food Engineering, v. 36, p. 81-87, 1998.

MACHADO, M. F.; OLIVEIRA, F. A. R. \& CUNHA, L. M. Effect of milk fat and total solids concentration on the kinetics of moisture uptake by ready-to-eat breakfast cereal. International Journal of Food Science and technology, v. 34, n. 1, p. 47-57, 1999.

MARQUES, L. G.; FERREIRA, M. C.; FREIRE, J. T. Freeze-drying of acerola (Malpighia glabra L.). Chemical Engineering and Processing: Process Intensification, v. 46, n. 5, p. 451-457, 2007.

MARQUES, L. G.; FERREIRA, M. C.; FREIRE, J. T. Freeze-drying of acerola (Malpighia glabra L.), Chemical Engineering and Processing, v 46, p.451457, 2007.

MARQUES, L. G.; SILVEIRA, A. M., FREIRE, J. T. Freeze-drying characteristics of tropical fruits, Drying Technology, v. 24(1-7), p. 457-463, 2006.

MARQUES, L.G. Liofilização de frutas tropicais, Tese de Doutorado em Engenharia Química, Universiade Federal de São Carlos, São Carlos, 2008.

MASON, T. J. Power ultrasound in food processing - the way forward. Thomson Science., London, UK, p. 105-126, 1998.

RATTI, C. Hot air and freeze-drying of highvalue foods: a review, Journal of Food Engineering, v.49, p. 311-319, 2001.

SANTOS, L. D. M., MARQUES, L. G., PRADO, M. M., 2011. Estudo comparativo de técnicas de secagem para sementes de Moringa oleífera L. ENEMP. 2011.
SOGI, D. S.; SIDDIQ, M.; DOLAN, K. D. Total phenolics, carotenoids and antioxidant properties of Tommy Atkin mango cubes as affected by drying techniques. LWT - Food Science and Technology, v. 62, n. 1, p. 1-5, 2014.

\section{AGRADECIMENTOS}

Os autores agradecem a CAPES e COPES/UFS pelo auxílio financeiro. 